\title{
Preface to JAISE 12(4)
}

\author{
Vincent Tam ${ }^{\mathrm{a}}$, Hamid Aghajan ${ }^{\mathrm{b}}$, Andrés Muñoz ${ }^{\mathrm{c}}$ and Juan Carlos Augusto ${ }^{\mathrm{d}}$ \\ ${ }^{a}$ Department of Electrical and Electronic Engineering, The University of Hong Kong, Hong Kong \\ ${ }^{\mathrm{b}}$ imec, IPI, Department of Telecommunications and Information Processing, Ghent University, Ghent, Belgium \\ ${ }^{c}$ Polytechnic School, Universidad Católica de Murcia, Spain \\ ${ }^{\mathrm{d}}$ Department of Comp. Science and Group on Development of Intelligent Environments, Middlesex University, \\ London, $U K$
}

\section{This issue}

This issue of JAISE is composed of five papers. The review process for the papers in this issue was supervised by our editors Davy Preuveneers, Andres Muñoz, and Vincent Tam, whom we thank for their service.

Internet of Things (IoT) technologies have drastically reshaped the whole world in various aspects including applications on agriculture, healthcare, smart cities and transportation. Most of these applications often involve a large number of sensors and devices producing a vast amount of real-time data for which location- and spatial-aware characteristics of sensing devices should be duly considered for useful analytics. Accordingly, indexing is one of the conventional ways to potentially minimize the response time of a query on the huge volume of real-time geospatial data involved in such applications. The paper "Indexing of real time geospatial data by IoT enabled devices: Opportunities, challenges and design considerations" by Chaudhry et al. conducts a detailed survey of the relevant literature on indexing real-time geospatial data generated by IoT enabled devices to carefully examine the key challenges related to the indexing of moving objects, and various critical design considerations. In this way, the paper enables researchers to better understand the principles, methods and challenges involved in the indexing of real-time geospatial data, thus aiding to identify new research opportunities in the nextgeneration IoT or smart city applications.

Ambient intelligence, communication and sensing technologies have been seamlessly integrated in many living environments as Ambient Assisted Living (AAL) systems to enhance the quality of life including personal healthcare in smart home environments in- stalled with motion and resource monitoring sensors. Most activity models deployed by the existing AAL systems rely on supervised learning classifiers to learn and recognize a fixed number of user activities with the available sensor data collected from the living environments. This will surely restrict the applicability of existing AAL systems as the constructed activity models cannot be flexibly extended to cater for new user activities. The paper "Evolving models for incrementally learning emerging activities" by Ye and Callus proposes an interesting technique of continual learning (CL) in machine learning to evolve an activity model over time with new types of activities for enhancing existing AAL systems. The involved technique notably combines two recent advances in CL, including the Net2Net technique to accelerate learning via knowledge transfer, and the Gradient Episodic Memory to alleviate forgetting while supporting beneficial transfer of knowledge to the previously learned activities. The resulting CL framework has been carefully evaluated on two real-world data sets to enhance its learning capability for accommodating incrementally introduced new user activities while retaining the accuracy of recognizing all the previous activities.

In addition to the installation of sensors for monitoring user activities or resource consumption in smart environments, smartphones readily equipped with a diversity of location, image and motion sensors are very convenient and powerful choices for continuously capturing users' behavioural data anytime and anywhere for further analyses. The paper "Personal productivity monitoring through smartphones" by Khan et al. proposes an approach to enhance individual's productivity by unobtrusively learning their routines through a smartphone application to periodically collect users' behavioural data such as the total amount of time spent 
at home or workplace during the study. Correlation analysis is performed on the collected behavioural data and user's productivity scores, surveyed from 10 users over 15 days, to extract significant behavioural features for training supervised machine learning algorithms like support vector machines to predict the productivity scores by analysing the changes in behavioural data. To further evaluate the effectiveness of the proposal, the publicly available Aruba dataset of a smart home project has been used to predict the productivity of the home occupants.

After work, many people may spend leisure time with family and friends to explore cultural and historical facts through visiting exhibition centres and museums. Most members of such small-group visits tend to have diverse preferences for which both individual and group preferences should be duly considered to construct a cohesive and harmonious tour path. This is where a smart environment system can offer help. The paper "Harmonizing divergent user preferences for cultural enrichment of small group visit" by Shin and Yoon proposes a mixed-initiative path planning system for a small group visit. The proposed path planning system can automatically merge individual profiles and then generate an intermediate path when most of the users' preferences are similar. Otherwise, the users will be asked to decide their path when their preferences are divergent. The proposed path planning system for small group visits is critically evaluated for its performance on a synthesized group simulation and also a comparative user study.

In many smart living or work platforms, sensors and Internet of Things technologies play a crucial role in building a smart data logger system to continuously measure and monitor ambient parameters such as air pressure and temperature for intelligent control of the environment. The paper "A smart data logger system based on sensor and Internet of Things technology as part of the smart faculty" by Djordjević et al. considers a reconfigurable smart data logger as a part of a smart faculty system to measure, monitor and then control ambient parameters so as to achieve optimal working conditions for an amphitheatre during examinations and preparatory classes in a college. In addition to the main control unit to monitor the collected sensor data, control nodes are activated to determine ambient parameters such as lighting, window blinds, and ventilation. Monitoring of the measured ambient parameters is also possible with a smartphone application that can be used to control the relevant ambient parameters as well as those of additional data logger systems.

\section{Upcoming issues}

The following is the list of upcoming issues of JAISE:

- September 2020: Thematic Issue on "Smart Environments and Ambient Intelligence in Agricultural and Environmental Technology"

- November 2020: Regular Issue

- January 2021: Thematic Issue on "Deep Learning-based Real-time Visual Analytics in Smart City"

- March 2021: Regular Issue

- May 2021: Thematic Issue on "Trustworthy Computing for Secure Smart Cities"

- July 2021: Regular Issue

More information on the call for papers for future thematic issues is available on the webpage of JAISE at: http://www.iospress.nl/journal/journal-of-ambientintelligence-and-smart-environments/ 